\title{
Vacuum tunneling and periodic structure in lattice Higgs models
}

\author{
F. Karsch ${ }^{\mathrm{a}, \mathrm{b}}$, M.L. Laursen ${ }^{\mathrm{a}, \mathrm{b}}$, T. Neuhaus ${ }^{\mathrm{b}}$ and B. Plache ${ }^{\mathrm{b}}$ \\ ${ }^{a}$ HLRZ, c / o KFA Jülich, P.O. Box 1913, D-52425 Jülich, Germany \\ ${ }^{b}$ Fakultät für Physik, Universität Bielefeld, P.O. Box 100131, D-33501 Bielefeld, Germany
}

\begin{abstract}
Using a geometric definition for the lattice Chern-Simons term in even dimensions, we have studied the distribution of Chern-Simons numbers for the $2 \mathrm{~d} U(1)$ and the $4 \mathrm{~d} S U(2)$ lattice Higgs models. The periodic structure of the distributions is preserved in our lattice formulation and has been examined in detail. In both cases the finite-size effects visible in the distribution of Chern-Simons numbers are well accounted for by the Haar measure. Moreover, we find that $\left\langle N_{\mathrm{CS}}^{2}\right\rangle$ grows with the spatial volume. We also find numerical evidence that tunneling in $4 \mathrm{~d}$ is increased at high temperature.
\end{abstract}

\section{Introduction}

Recent interest in the finite-temperature electroweak phase transition has focused on the role of tunneling between topologically distinct vacuums. Such tunnelings, in a semiclassical approximation, are well known in the continuum and lead to a periodic structure in the effective potential [1]. During such a tunneling the so-called topological charge will change by an integer. In two dimensions there are the kinks, which are time-independent finite-energy solutions of the scalar Higgs model or the sine-Gordon model. The vacuum solutions occur at spatial infinity and the tunneling is between $\phi(x=-\infty)$ and $\phi(x=+\infty), \phi$ being the Higgs field. In the $2 \mathrm{~d}$ abelian gauge Higgs system the tunneling is in euclidean time and governed by time-dependent finite-energy solutions - the vortices. The tunneling is most transparent if one assumes an axial gauge $A_{0}=0$. The vacuum gauge field at temporal infinity becomes pure gauge $A_{i}(t, x)=\phi(t, x)^{-1} \partial_{i} \phi(t, x)$ and the tunneling goes from $A_{i}(t=-\infty, x)$ to $A_{i}(t=+\infty, x)$. In four dimensions one has instantons in the pure SU(2) gauge theory. This case is very similar to the previous one, upon replacing the Higgs field with a proper gauge transformation. With Higgs fields included no time-dependent finite-energy solutions are known. However, assuming static fields only, one has a saddle-point solution - the sphaleron [2]. The sphaleron in itself does not provide the tunneling. It is so to 
speak lying midways between two vacuums and we must still imagine a time-dependent field configuration interpolating among the two vacuums.

The physical relevance of these tunnelings was pointed out by 't Hooft who found that neither baryon nor lepton numbers are conserved in the electroweak theory [3]. For the baryon and lepton currents one has

$$
\partial_{\mu} J_{\mu}^{B}=\partial_{\mu} J_{\mu}^{\mathrm{e}}=\frac{N_{\mathrm{f}}}{16 \pi^{2}} \operatorname{tr}\left[F_{\mu \nu} \tilde{F}_{\mu \nu}\right]
$$

where $N_{\mathrm{f}}$ is the number of families of quarks and leptons. While the $B-L$ symmetry remains unbroken due to the anomaly cancellation, $B+L$ is no longer conserved. This so-called baryon number violation is caused by the non-trivial topological winding of the SU(2) gauge fields. The baryon number $B$ changes by an amount

$$
\Delta B=\frac{\underline{N_{f}}}{16 \pi^{2}} \int_{t_{1}}^{t_{2}} \mathrm{~d} t \int \mathrm{d}^{3} x \operatorname{tr}\left[F_{\mu \nu} \tilde{F}_{\mu \nu}\right] .
$$

In the axial gauge, $A_{0}=0, \Delta B$ is related to the change in the Chern-Simons (CS) number $N_{\mathrm{CS}}$,

$$
\Delta B=N_{\mathrm{f}}\left[N_{\mathrm{CS}}\left(t_{2}\right)-N_{\mathrm{CS}}\left(t_{1}\right)\right]
$$

with

$$
N_{\mathrm{CS}}=-\frac{1}{8 \pi^{2}} \int \mathrm{d}^{3} x \epsilon_{i j k} \operatorname{tr}\left[A_{i}\left(\partial_{j} A_{k}+\frac{2}{3} A_{j} A_{k}\right)\right]
$$

At zero temperature only quantum tunneling via instanton-like configurations is possible. The rate for such a process is, however, exponentially suppressed. This is because the relevant field configurations have an action of the order of the barrier height $2 \pi / \alpha_{\mathrm{W}}$, with $\alpha_{\mathrm{W}}$ denoting the electroweak coupling constant. At high temperatures such an exponential suppression is absent, since tunneling can occur classically by thermal fluctuations. Assuming that the temperature is so high that only static fields (kinds or sphalerons) are relevant, one can go over to a hamiltonian formulation of the theory and study the evolution of the system in real time via the classical hamiltonian equation of motion or the Langevin equations with a friction term. Classical nucleation theory can then be applied to extract information about the rate of baryon number violating processes. This has been done for the above-mentioned lattice models [4-7]. However, a priori it is not known whether the static field approximation is valid and we, therefore have attempted to study the full theory in euclidean time. By varying the time extent of the lattice we can in principle control the temperature. We have initiated work in the $2 \mathrm{~d}$ abelian gauge Higgs model and the $4 d \mathrm{SU}(2)$ gauge Higgs model [8]. 
In two dimensions it is easy to derive an expression for the Chern-Simons number, $N_{\mathrm{CS}}$, on the lattice, it amounts to evaluating the sum of the link angles in a Polyakov line. In four dimensions this is much more involved. There are several geometric definitions of $N_{\mathrm{CS}}$ [9-12]. We decided to use the version by Seiberg [10], which shares the properties of the continuum expression. It is gauge dependent and changes by an integer under large gauge transformations. We note that $N_{\mathrm{CS}}$ is in general non-integer; only for configurations which behave as pure gauge it is indeed an integer. Numerical investigations of topological properties of lattice gauge theories are known to be difficult and time consuming, if one attempts to preserve the important quantization properties of topological objects also on the lattice. In the geometrical approaches an interpolation of the original gauge fields into the elementary cells of the $d$-dimensional lattice is required and a $d$-dimensional integration over the fields needs to be performed. Starting from an existing program for the calculation of topological charges [11] we developed a program for the calculation of Chern-Simons numbers, which is fully vectorized and very efficient.

A central goal in studies of CS numbers in Higgs models on euclidean lattices is the calculation of the distribution of CS numbers, $P\left(N_{\mathrm{CS}}\right)$, and the determination of the temperature dependence of the barrier height between topologically distinct vacuums. In the high-temperature limit, where static field configurations dominate, this is related to the difference between the minima and maxima of the effective potential, $V\left(N_{\mathrm{CS}}\right)$, for the CS numbers, which can be extracted from the probability distribution,

$$
\begin{aligned}
P\left(N_{\mathrm{CS}}\right)= & \exp \left(-V\left(N_{\mathrm{CS}}\right)\right) \\
= & \int \mathrm{d} A \mathrm{~d} \phi \exp (-S(A, \phi)) \\
& \times \delta\left(N_{\mathrm{CS}}+\frac{1}{8 \pi^{2}} \int \mathrm{d}^{3} x \epsilon_{i j k} \operatorname{tr}\left[A_{i}\left(\partial_{j} A_{k}+\frac{2}{3} A_{j} A_{k}\right)\right]\right) .
\end{aligned}
$$

However, it also is known that even in the continuum at zero temperature, vacuum fluctuations may lead to rather large values of the CS numbers and may easily dominate the above distribution and cover any expected periodic structure of the potential expected to arise from topological properties of the field configurations [5-7]. On the lattice one encounters in addition the problem of finding a formulation for the CS numbers which correctly reproduces the topological properties known in the continuum. It is the purpose of this paper to systematically investigate these problems on the lattice. We will study the properties of a lattice version of the CS term suggested by Seiberg [10]. 
The outline of the paper is as follows. In sect. 2 we discuss topological charge and Chern-Simons numbers in the continuum. In sect. 3 we give the definitions of the lattice topological charge and Chern-Simons term. In sect. 4 we derive expressions for the distribution of $N_{\mathrm{CS}}$ in two dimensions at strong coupling and we present results for the periodic structure. Sect. 5 is devoted to our results in four dimensions, and we describe the algorithm used in our simulations of the SU(2) Higgs model. Finally we give our conclusions in sect. 6.

\section{Topological charge and the Chern-Simons term in the continuum}

We will first consider the $2 \mathrm{~d} U(1)$ case. It is well known that one can define a gauge-invariant topological charge $Q$ :

$$
\begin{aligned}
Q & =-\frac{1}{4 \pi} \int_{\mathrm{M}} \mathrm{d} t \mathrm{~d} x \epsilon_{\mu \nu} F_{\mu \nu} \in \mathbb{Z} . \\
F_{\mu \nu} & =\partial_{\mu} A_{\nu}-\partial_{\nu} A_{\mu} .
\end{aligned}
$$

The manifold is denoted $M$ and we will assume that its boundary $\partial M$ is a one-sphere $\mathrm{S}^{1}$. The topological charge density, $q$, can be written as a divergence of the Chern-Simons density $K_{\mu}$,

$$
\begin{aligned}
q & =-\frac{1}{4 \pi} \epsilon_{\mu \nu} F_{\mu \nu}=\partial_{\mu} K_{\mu}, \\
K_{\mu} & =-\frac{1}{2 \pi} \epsilon_{\mu \nu} A_{\nu} .
\end{aligned}
$$

Under a local gauge transformation, $g$, the gauge field, $A_{\mu}$, changes like

$$
\delta A_{\mu}=\frac{1}{i} g^{-1} \partial_{\mu} g
$$

so that

$$
\delta K_{\mu}=-\frac{1}{2 \pi i} \epsilon_{\mu \nu} \partial_{\nu} g g^{-1}
$$

We define the Chern-Simons number $N_{\mathrm{CS}}$ at a given euclidean time as follows:

$$
N_{\mathrm{CS}}=\int_{\partial \mathrm{M}} \mathrm{d} x K_{0}
$$


We note that $N_{\mathrm{CS}}$ is an integer only for pure gauge (vacuum) configurations and is gauge dependent. It changes, however, under a gauge transformation only by an integer

$$
\delta N_{\mathrm{CS}}=-\frac{1}{2 \pi i} \int_{\partial \mathrm{M}} \mathrm{d} x \partial_{1} g g^{-1} \in \mathbb{Z}
$$

This follows also from homotopy theory using the mapping $g: S^{1} \rightarrow U(1)=S^{1}$. Such mappings are characterized by the homotopy class $\Pi_{1}\left(\mathrm{~S}^{1}\right) \in \mathbb{Z}$. In the tunneling picture vortices will interpolate between two vacuums with integer $N_{\mathrm{CS}}=n, n+1$.

In the $4 \mathrm{~d} \mathrm{SU}(2)$ case the topological charge $Q$ is

$$
\begin{aligned}
Q & =-\frac{1}{32 \pi^{2}} \int_{\mathrm{M}} \mathrm{d} t \mathrm{~d}^{3} x \epsilon_{\mu \nu \rho \sigma} \operatorname{tr}\left[F_{\mu \nu} F_{\rho \sigma}\right] \in \mathbb{Z}, \\
F_{\mu \nu} & =\partial_{\mu} A_{\nu}-\partial_{\nu} A_{\mu}+\left[A_{\mu}, A_{\nu}\right] .
\end{aligned}
$$

Here we shall assume that $\partial \mathrm{M}=\mathrm{S}^{3}$. Like in two dimensions we can write

$$
\begin{gathered}
q=-\frac{1}{32 \pi^{2}} \epsilon_{\mu \nu \rho \sigma} \operatorname{tr}\left[F_{\mu \nu} F_{\rho \sigma}\right]=\partial_{\mu} K_{\mu}, \\
K_{\mu}=-\frac{1}{8 \pi^{2}} \epsilon_{\mu \nu \rho \sigma} \operatorname{tr}\left[A_{\nu}\left(\partial_{\rho} A_{\sigma}+\frac{2}{3} A_{\rho} A_{\sigma}\right)\right] .
\end{gathered}
$$

Under a local gauge transformation, $g$, the gauge field changes as

$$
\delta A_{\mu}=g^{-1}\left[A_{\mu}+\partial_{\mu}\right] g
$$

giving

$$
\begin{aligned}
\delta K_{\mu}= & -\frac{1}{24 \pi^{2}} \epsilon_{\mu \nu \rho \sigma} \operatorname{tr}\left[\partial_{\nu} g g^{-1} \partial_{\rho} g g^{-1} \partial_{\sigma} g g^{-1}\right] \\
& -\frac{1}{8 \pi^{2}} \epsilon_{\mu \nu \rho \sigma} \partial_{\nu} \operatorname{tr}\left[\partial_{\rho} g g^{-1} A_{\sigma}\right]
\end{aligned}
$$

The (timelike) Chern-Simons number $N_{\mathrm{CS}}$ is then defined exactly as in eq. (2.5), and has the same properties. Its gauge variation in an integer (the boundary term vanishes)

$$
\delta N_{\mathrm{CS}}=-\frac{1}{24 \pi^{2}} \epsilon_{0 \nu \rho \sigma} \int_{\partial \mathrm{M}} \mathrm{d}^{3} x \operatorname{tr}\left[\partial_{\nu} g g^{-1} \partial_{\rho} g g^{-1} \partial_{\sigma} g g^{-1}\right] \in \mathbb{Z} .
$$


This time the mapping $g: \mathrm{S}^{3} \rightarrow \mathrm{SU}(2)=\mathrm{S}^{3}$, and the homotopy class is $\Pi_{3}\left(\mathrm{~S}^{3}\right) \in \mathbb{Z}$. In this case the instanton will interpolate between two vacuums, assuming the axial gauge.

\section{Topological charge and the Chern-Simons term on the lattice}

We will now consider the lattice version of the topological charge and the Chern-Simons number. We will use a geometric definition given by Seiberg [10]. Problems with dislocations will be ignored here. It is convenient to start from the definition of the topological charge first given by Lüscher [9]. The following considerations apply to $2 \mathrm{~d}$ or $4 \mathrm{~d}$. The manifold $\mathrm{M}$ is a torus and we will cover it with cells $\mathrm{c}(n)$, where $n$ denotes the lattice sites. Let the gauge potential $A_{\nu}^{n}$ be defined on $\mathrm{c}(n)$ and likewise $A_{\nu}^{n-\hat{\mu}}$ be defined on $\mathrm{c}(n-\hat{\mu})$. At the faces $\mathrm{f}(n, \mu)=$ $\mathrm{c}(n-\hat{\mu}) \cap \mathrm{c}(n)$, we can relate the two potentials by a transition function $v_{n, \mu}(x)$,

$$
A_{\nu}^{n-\hat{\nu}}(x)=v_{n, \mu}^{-1}(x)\left[A_{\nu}^{n}(x)+\partial_{\nu}\right] v_{n, \mu}(x)
$$

At the corners of the faces the transition function is given by

$$
v_{n, \mu}(x)=w^{n-\mu}(x) w^{n}(x)^{-1}
$$

Here $w^{n}(x)$ is a parallel transporter, used to gauge fix the links to the complete axial gauge in each cell. By interpolation this formula is extended to the whole face. The transition function, $v_{n, \mu}(x)$, defines a bundle, while $w^{n}(x)$ (given on the boundary of the cell) is a section of the bundle [13]. In Lüscher's approach [9] the interpolating fields in the interior of the cells are given by

$$
\begin{aligned}
& v_{n, \mu}(x)=s_{n, \mu}^{n-\mu}(x)^{-1} v_{n, \mu}(n) s_{n, \mu}^{n}(x), \\
& s_{n, \mu}^{t}(x)=w^{t}(n) S_{n, \mu}(x) w^{t}(x)^{-1}, \quad t=n, n-\mu .
\end{aligned}
$$

From now on we shall assume that $t=n$ or $t=n-\mu$ and that $\nu \neq \mu$. In $2 \mathrm{~d}$ one has explicitly

$$
\begin{aligned}
s_{n, \mu}^{t}(x) & =\left[w^{t}(n) U(n, \nu) w^{t}(n+\nu)^{-1}\right]^{x}, \\
w^{t}(x) & =\left[w^{t}(n) w^{t}(n+\nu)^{-1}\right]^{x} w^{t}(n), \quad \nu \neq \mu, \quad t=n, n-\mu, \\
S_{n, \mu}(x) & =U(n, \nu)^{x} .
\end{aligned}
$$


The approach of Seiberg is quite similar [10]. He does not apply any axial gauge fixing, and his function $S_{n, \mu}$ depends on the original fields. However, otherwise the interpolation formulas for $s_{n, \mu}^{t}(x)$ and $S_{n, \mu}(x)$ are identical. Although the $4 \mathrm{~d}$ case is more involved the previous statement holds as well. For the Lüscher charge [9] one has

$$
\begin{aligned}
Q^{\mathrm{L}} & =\sum_{n} q^{\mathrm{L}}(n)=\sum_{n, \mu}(-1)^{\mu}\left(k_{n, \mu}-k_{n+\mu, \mu}\right), \quad\left|q^{\mathrm{L}}(n)\right| \leqslant \frac{1}{2}, \\
(-1)^{\mu} k_{t, \mu} & =-\frac{1}{2 \pi i} \epsilon_{\mu \nu} \int_{f(t, \mu)} \mathrm{d} x s_{t, \mu}^{n}(x)^{-1} \partial_{\nu} s_{t, \mu}^{n}(x) .
\end{aligned}
$$

Notice that $k_{t, \mu}$ is gauge invariant. After a little algebra one finds $q^{\mathrm{L}}(n)=\frac{1}{2 \pi i} \log \left[U(n, 1) U(n+1,2) U(n+2,1)^{-1} U(n, 2)^{-1}\right], \quad\left|q^{\mathrm{L}}(n)\right| \leqslant \frac{1}{2}$.

The Seiberg charge [10] is obtained by replacing

$$
\left[s_{t, \mu}^{n}(x), k_{t, \mu}\right] \rightarrow\left[S_{t, \mu}(x), K_{t, \mu}\right]
$$

where $K_{t, \mu}$ can now be interpreted as the local Chern-Simons term.

$$
\begin{aligned}
Q^{\mathrm{S}} & =\sum_{n} q^{\mathrm{S}}(n)=\sum_{n, \mu}(-1)^{\mu}\left(K_{n, \mu}-K_{n+\mu, \mu}\right), \quad\left|q^{\mathrm{S}}(n)\right| \leqslant \frac{1}{2}, \\
(-1)^{\mu} K_{t, \mu} & =-\frac{1}{2 \pi i} \epsilon_{\mu \nu} \int_{f(t, \mu)} \mathrm{d} x S_{t, \mu}(x)^{-1} \partial_{\nu} S_{t, \mu}(x) .
\end{aligned}
$$

Though each $K_{n, \mu}$ term is gauge dependent, the charge remains gauge invariant, in fact $q^{\mathrm{S}}(n)=q^{\mathrm{L}}(n)$. This follows from the relation

$$
\begin{aligned}
q^{\mathrm{S}}(n) & =q^{\mathrm{L}}(n)-q^{w}(n), \\
q^{w}(n) & =\frac{1}{2 \pi i} \epsilon_{\mu \nu} \int_{\partial \mathrm{c}(n)} \mathrm{d} x w^{n}(x)^{-1} \partial_{\nu} w^{n}(x) .
\end{aligned}
$$

The last piece is an integer since we integrate the section over the boundary of the cell. By restricting the charge to the interval $|q(n)| \leqslant \frac{1}{2}$, it follows that the two charge definitions agree. The Chern-Simons term for the $2 \mathrm{~d}$ U(1) Higgs model is now given by

$$
N_{\mathrm{CS}} \equiv \sum_{n_{\mathrm{s}}} K_{n_{\mathrm{s}}, \mu}=\frac{1}{2 \pi i} \sum_{n_{\mathrm{s}}} \log U\left(n_{\mathrm{s}}, \nu\right)
$$


The summation is only over the spatial lattice at a fixed euclidean time, i.e. $N_{\mathrm{CS}} \equiv N_{\mathrm{CS}}(t)$. Under gauge transformations $N_{\mathrm{CS}}$ indeed changes by an integer. Moreover, it is an integer for pure gauge.

We can extend these considerations to the four-dimensional case. Defining for shortness

$$
\begin{aligned}
& \mathscr{S}_{\nu}^{t}(x)=s_{t, \mu}^{n}(x)^{-1} \partial_{\nu} s_{t, \mu}^{n}(x), \\
& \mathscr{P}_{\nu}^{t}(x)=p_{t+\nu, \mu \nu}^{n}(x)^{-1} \partial_{\nu} p_{t+\nu, \mu \nu}^{n}(x),
\end{aligned}
$$

one finds

$$
\begin{aligned}
Q^{\mathrm{L}}= & \sum_{n} q^{\mathrm{L}}(n)=\sum_{n, \mu}(-1)^{\mu}\left(k_{n, \mu}-k_{n+\mu, \mu}\right) \\
(-1)^{\mu} k_{t, \mu}= & -\frac{1}{24 \pi^{2}} \epsilon_{\mu \nu \rho \sigma} \int_{f(t, \mu)} \mathrm{d}^{3} x \operatorname{tr}\left[\mathscr{S}_{\nu}^{t}(x) \mathscr{S}_{\rho}^{t}(x) \mathscr{S}_{\sigma}^{t}(x)\right] \\
& +\frac{1}{8 \pi^{2}} \epsilon_{\mu \nu \rho \sigma} \int_{p(t+\nu, \mu, \nu)} \mathrm{d}^{2} x \operatorname{tr}\left[\mathscr{P}_{\rho}^{t}(x) \mathscr{S}_{\sigma}^{t}(x)\right] .
\end{aligned}
$$

The actual expressions for $s_{t, \mu}^{n}(x)$ and $p_{t+\nu, \mu \nu}^{n}(x)$ are given in ref. [9]. In Seiberg's version we use the same expressions but replace

$$
\left[s_{t, \mu}^{n}(x), p_{t+\nu, \mu \nu}^{n}(x), k_{t, \mu}\right] \rightarrow\left[S_{t, \mu}(x), P_{t+\nu, \mu \nu}(x), K_{t, \mu}\right] .
$$

The Seiberg charge will be gauge invariant, if we use the same restriction as in two dimensions, and the Chern-Simons term is given by $N_{\mathrm{CS}} \equiv \sum_{n_{\mathrm{s}}} K_{n_{\mathrm{s}}, \mu}$. In the naive continuum limit $a \rightarrow 0$ one finds with $U(n, \mu)=\exp \left(a A_{n, \mu}\right)$

$$
(-1)^{\mu} K_{t, \mu}=-\frac{a^{3}}{8 \pi^{2}} \epsilon_{\mu \nu \rho \sigma} \operatorname{tr}\left[A_{t, \nu}\left(\partial_{\rho} A_{t, \sigma}+\frac{2}{3} A_{t, \rho} A_{t, \sigma}\right)\right]
$$

It is easy to show that

$$
K_{t, \mu}=k_{t, \mu}+K_{t, \mu}^{w}
$$

where $K_{t, \mu}^{w}$ has the same form as $K_{t, \mu}$, but with $S_{t, \mu}(\mathrm{x}) \rightarrow w^{t}(x)$. As the section transforms like a gauge transformation this implies that the Chern-Simons number will change by an integer. For pure gauge fields one finds in addition $s_{t, \mu}^{n}(x)=1$ and $S_{t, \mu}(x)=P_{t+\nu, \mu \nu}(x)$ at the plaquette. We, therefore, find

$$
(-1)^{\mu} K_{t, \mu}=-\frac{1}{24 \pi^{2}} \epsilon_{\mu \nu \rho \sigma} \int_{f(t, \mu)} \mathrm{d}^{3} x \operatorname{tr}\left[\mathscr{W}_{\nu}^{n} \mathscr{W}_{\rho}^{n} \mathscr{W}_{\sigma}^{n}\right], \quad \mathscr{W}_{\nu}^{n}=w^{n}(x)^{-1} \partial_{\nu} w^{n}(x) .
$$


This expression closely resembles the continuum form. After summation over the spatial volume $N_{\mathrm{CS}}$ indeed becomes an integer even on finite lattices.

We finally note that the topological charges, obtained from the two approaches discussed above, are related [14]. Using eqs. (3.2) and (3.5) one has for any configuration

$$
\begin{aligned}
& q^{\mathrm{S}}(n)=q^{\mathrm{L}}(n)-q^{w}(n) \\
& q^{w}(n)=\frac{1}{24 \pi^{2}} \epsilon_{\mu \nu \rho \sigma} \int_{\partial \mathrm{c}(n)} \mathrm{d}^{3} x \operatorname{tr}\left[\mathscr{W}_{\nu}^{n} \mathscr{W}_{\rho}^{n} \mathscr{W}_{\sigma}^{n}\right] .
\end{aligned}
$$

Here, $q^{w}(n)$ is the topological charge (integer) of the section and $Q^{\mathrm{L}}=Q^{w}$. Notice, that there is no restriction on $q^{\mathrm{L}}(n)$ so that $q^{\mathrm{S}}(n)=q^{\mathrm{L}}(n)$ up to integers. For smooth fields like instantons they always agree, while for realistic field configurations this is true for almost every cell.

\section{Chern-Simons number in the strong-coupling limit, finite-size effects}

We will discuss here the distribution of CS numbers induced by the Haar measure on finite lattices, i.e. in the absence of any Boltzmann weight factors in the euclidean path integral over configuration space. We will call this the strongcoupling limit. For the U(1) Higgs model in two dimensions it is easy to give an expression for the distribution of Chern-Simons numbers in this limit. Using the definition given in eq. (3.10) one finds on a lattice with spatial extent $n$ the following recursive relation for the density, $\rho_{n}(z)$, of CS numbers $z$,

$$
\begin{gathered}
\rho_{1}(z)= \begin{cases}1 & |z| \leqslant \frac{1}{2} \\
0 & \text { otherwise, }\end{cases} \\
\rho_{n}(z)=\prod_{i=1}^{n} \int_{-1 / 2}^{1 / 2} \mathrm{~d} z_{i} \rho_{1}\left(z_{i}\right) \delta\left(\sum_{j=1}^{n} z_{j}-z\right)=\int_{\max ((1-n) / 2, z-1 / 2)}^{\min ((n-1) / 2, z+1 / 2)} \mathrm{d} z \rho_{n-1}(z) .
\end{gathered}
$$

Using this recursion formula we find explicitly,

$$
\begin{gathered}
\rho_{2 n+1}(z)=\frac{1}{(2 n) !} \sum_{i=0}^{n-m}(-1)^{i}\left(\begin{array}{c}
2 n+1 \\
i
\end{array}\right)\left(n+\frac{1}{2}-i-|z|\right)^{2 n} \\
m-\frac{1}{2} \leqslant|z| \leqslant m+\frac{1}{2} \\
\rho_{2 n}(z)=\frac{1}{(2 n-1) !} \sum_{i=0}^{n-1-m}(-1)^{i}\left(\begin{array}{c}
2 n \\
i
\end{array}\right)(n-i-|z|)^{2 n-1} \\
m \leqslant|z| \leqslant m+1
\end{gathered}
$$


These relations allow us to define moments of the distributions as

$$
\mathscr{P}_{n}^{\alpha}=\int_{-n / 2}^{n / 2} \mathrm{~d} z z^{\alpha} \rho_{n}(z)
$$

Some properties of $\rho_{n}(z)$ and these moments are discussed in the appendix. In particular it is obvious that all odd moments vanish and $\rho_{n}(z) / \rho_{n}(0) \rightarrow 1$ for $n \rightarrow \infty$. However, on a lattice of finite extent $n$, the distribution functions, $\rho_{n}$, are close to gaussians, i.e. large values of the CS numbers are suppressed due to finite-size effects. This is reflected in the even moments,

$$
\begin{aligned}
& \mathscr{P}_{n}^{0}=1, \\
& \mathscr{P}_{n}^{2}=\frac{n}{12}, \\
& \mathscr{P}_{n}^{4}=-\frac{n}{120}+\frac{n^{2}}{48} .
\end{aligned}
$$

The leading term for $\mathscr{P}_{n}^{2 \alpha}=(2 \alpha-1) ! !\left(\mathscr{P}_{n}^{2}\right)^{\alpha}$ is just coming from a gaussian approximation *. Therefore $\left\langle N_{\mathrm{CS}}^{2}\right\rangle$ grows with the number of links in a timeslice. Notice the fourth-order cumulant $\mathscr{P}_{n}^{4}-3\left(\mathscr{P}_{n}^{2}\right)^{2}=-n / 120$.

We will mainly be interested in the gauge-invariant, non-integer part of the CS numbers. This can be obtained by summing over the various gauge equivalent sectors of the distribution functions. On a lattice with $(2 n+1)$ sites in spatial direction these are given by (see eq. (A.1) for even number of lattice sites)

$$
R_{2 n+1}^{\alpha}(z)=\sum_{m=-n}^{n}(z+m)^{\alpha} \rho_{2 n+1}(z+m), \quad|z| \leqslant \frac{1}{2} .
$$

We show in the appendix that on a lattice of size $n$ the distribution $R_{n}^{0}$ and the moments $R_{n}^{\alpha}$ are, in fact, independent of $z$ in the strong-coupling limit, and equal the moments $\mathscr{P}_{n}^{\alpha}$. In particular, this means that $R_{n}^{0}(z) \equiv 1$ for all $z$, i.e. the distribution of the non-integer part of the CS numbers is flat in configuration space even on finite lattices.

Let us now discuss some numerical results obtained in the strong-coupling regime. We used a $2 \mathrm{~d} U(1)$ lattice Higgs model. The lattice action is

$$
\begin{aligned}
S= & -\frac{\beta}{r} \sum_{n, \mu<\nu} \operatorname{tr}\left(U_{n, \mu \nu}\right)+\lambda \sum_{n}\left(\frac{1}{r} \operatorname{tr}\left(\Phi_{n}^{\dagger} \Phi_{n}\right)-1\right)^{2} \\
& -\frac{2 \kappa}{r} \sum_{n, \mu} \operatorname{tr}\left(\Phi_{n}^{\dagger} U_{n, \mu} \Phi_{n+\mu}\right)+\frac{1}{r} \sum_{n} \operatorname{tr}\left(\Phi_{n}^{\dagger} \Phi_{n}\right),
\end{aligned}
$$

\footnotetext{
* The second moment has previously been evaluated in ref. [15].
} 
with $r=1$ for $\mathrm{U}(1)$ and $r=2$ for SU(2). We studied the distribution of CS numbers for various parameter sets on lattices of size $12^{2}$ and $24^{2}$. In the left column of fig. 1 we show three distributions of the CS numbers obtained from calculations on the $12^{2}$ lattice at $(\beta, \lambda)=(2.0, \infty)$ and $\kappa=(0.5,2.0,8.0)$. At each of the $\kappa$-values 100.000 measurements of the CS number, defined by eq. (3.10), in each of the 12 time slices has been performed. The distributions for all 12 slices have then been added in order to increase the statistics.

The $\beta$-value has been chosen such that our strong-coupling calculations should be applicable, whenever the Higgs sector decouples from the gauge field sector. This happens at small values of $\kappa$. Indeed, no periodic structure is visible in the distribution for our lowest value of $\kappa$ and the distribution is well described by our analytic result, eq. (4.3). At large values of $\kappa$, however, the periodic structure becomes transparent. More and more of the configurations are close to pure gauge, hence the clustering of $N_{\mathrm{CS}}$ around integers. We note, however, that the peak heights are distributed according to the characteristic gaussian form of the strong-coupling distribution function. In the right column of fig. 1 we have divided

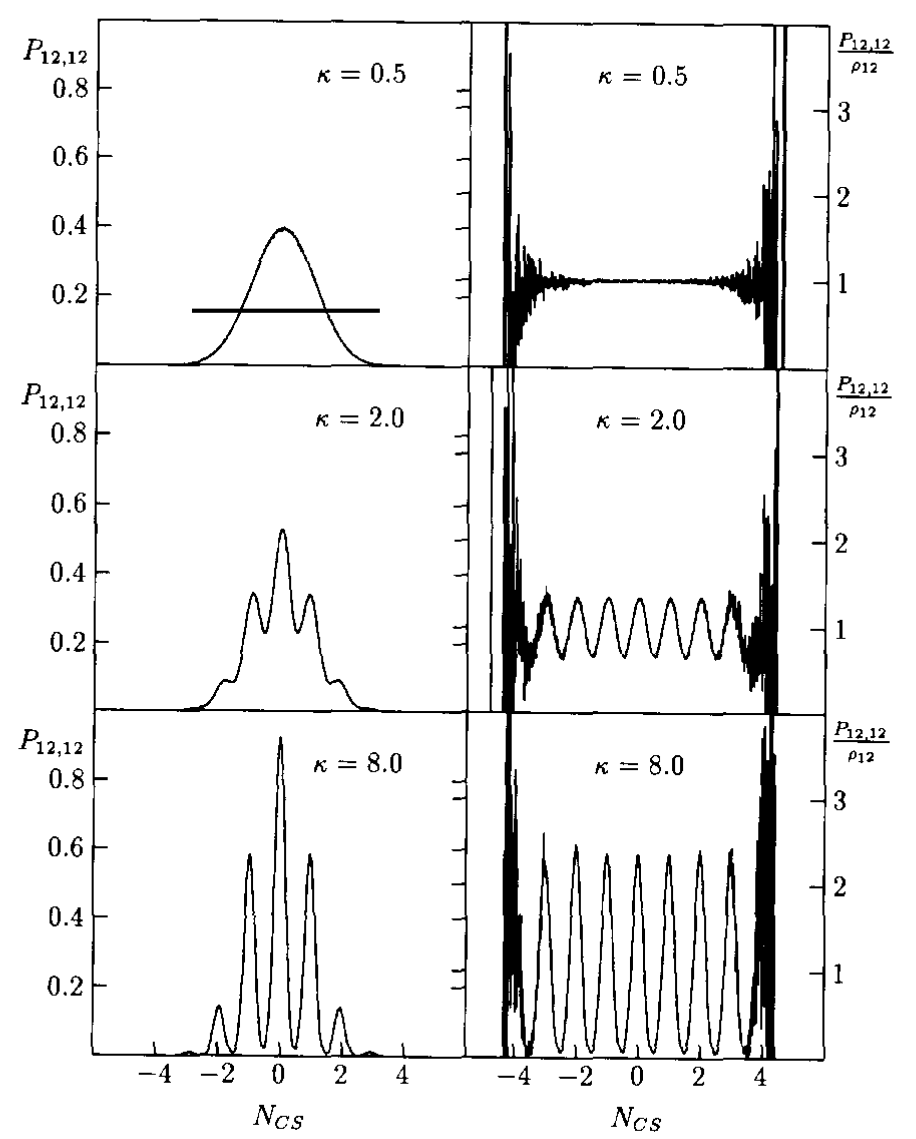

Fig. 1. Probability distributions for Chern-Simons numbers on a $12^{2}$ lattice. The left column shows the distributions obtained from simulations at $(\beta, \lambda)=(2.0, \infty)$ and the three $\kappa$-values given in the figure. The right column shows the same distributions divided by the strong-coupling distribution at $\beta=\kappa=0$ (eq. (4.3)). 
TABLE 1

Moments of the distribution of Chern-Simons numbers for the $2 \mathrm{~d} U(1)$ Higgs model on a $12^{2}$ lattice at $(\beta, \lambda)=(2.0, \infty)$. The last column shows the strong-coupling results given in eq. (4.5)

\begin{tabular}{ccccc}
\hline$\kappa$ & 0.5 & 2.0 & 8.0 & 0.0 \\
\hline$\left\langle N_{\mathrm{CS}}^{2}\right\rangle$ & $0.998(5)$ & $1.001(8)$ & $0.970(13)$ & 1.0 \\
$\left\langle N_{\mathrm{CS}}^{4}\right\rangle$ & $2.886(21)$ & $2.909(34)$ & $2.830(90)$ & 2.9 \\
\hline
\end{tabular}

the original distributions by the strong-coupling form of the distribution, $\rho_{12}(z)$, which reflects the non-uniform distributions of CS numbers in phase space due to finite lattice effects. The peak heights are now identical (The large fluctuations at higher $N_{\mathrm{CS}}$ values are due to limited statistics in this exponentially suppressed part of the distribution.). Although the shape of the distributions changes drastically, the moments of $N_{\text {CS }}$ are insensitive to this as can be seen from table 1. For each $\kappa$-value we find on the $12^{2}$ lattice $\left\langle N_{\mathrm{CS}}^{2}\right\rangle \simeq 1.00$ and $\left\langle N_{\mathrm{CS}}^{4}\right\rangle \simeq 2.90$, which is in perfect agreement with the exact results $\mathscr{P}_{12}^{2}=1$ and $\mathscr{P}_{12}^{4}=2.9$. Likewise for the $24^{2}$ lattice, $\left\langle N_{\mathrm{CS}}^{2}\right\rangle=2.09$ and $\left\langle N_{\mathrm{CS}}^{4}\right\rangle=11.80$ compared to $\mathscr{P}_{24}^{2}=2$ and $\mathscr{P}_{24}^{4}=11.8$. We also have checked that these results are independent of $\beta$. Hence, for large lattices the data are thus very well described by a gaussian distribution.

The results presented so far are in accordance with the assumption that the distribution of CS numbers on a lattice of size $n_{\sigma} \times n_{\tau}$ is a product of two probability distributions, where one is just given by $\rho_{n_{\sigma}}$ and describes the phasespace restrictions for $N_{\mathrm{CS}}$ and the other gives the probability to find a certain non-integer part, $z$, for the CS number, $N_{\mathrm{CS}}=m+z$. This part of the distribution is controlled by the action and thus contains the relevant physical information. Let us write the probability to find CS number $N_{\mathrm{CS}}=m+z$ as

$$
P_{n_{\sigma}, n_{\tau}}\left(N_{\mathrm{CS}}\right)=\omega_{n_{\sigma}, n_{\tau}}(z) \rho_{n_{\sigma}}(m+z), \quad|z| \leqslant \frac{1}{2},
$$

with $\rho_{n_{\sigma}}$ defined in eq. (4.3) and $\omega_{n_{r}, n_{\tau}}$ denoting a probability distribution that depends on the couplings of the euclidean action as well as the spatial and temporal size of the lattice. Using eq. (4.6) and (A.13) it is then easy to verify that indeed the moments, $\left\langle N_{\mathrm{CS}}^{\alpha}\right\rangle$, are independent of $\beta$ and $\kappa$.

TABLE 2

Moments of the distributions of the non-integer part $(z)$ of Chern-Simons numbers, $N_{\mathrm{CS}}=m+z$, for the $2 \mathrm{~d} U(1)$ Higgs model on a $12^{2}$ and $24^{2}$ lattices at $(\beta, \lambda)=(2.0, \infty)$

\begin{tabular}{ccccc}
\hline$\kappa$ & \multicolumn{2}{c}{$\underline{\left\langle z^{2}\right\rangle}$} & \multicolumn{2}{c}{$\left\langle z^{4}\right\rangle$} \\
\cline { 2 - 4 } & $n=12$ & $n=24$ & & $n=12$ \\
\hline 0.5 & $0.0832(1)$ & $0.0833(1)$ & $0.0125(1)$ & $0.0125(1)$ \\
2.0 & $0.0662(1)$ & $0.0805(1)$ & $0.0091(1)$ & $0.0119(1)$ \\
8.0 & $0.0287(1)$ & $0.0511(1)$ & $0.0024(1)$ & $0.0062(1)$ \\
\hline
\end{tabular}


Let us now discuss the distribution of the non-integer part of the CS number and the corresponding moments. These describe the fluctuations of the CS number around vacuum configurations in a given topological sector. In the continuum theory arguments have been given that these vacuum fluctuations are also proportional to the spatial volume of the system [6]. In table 2 we give results for the moments of the non-integer part of the CS numbers. We note that for a flat distribution one finds $\left\langle z^{2}\right\rangle=\frac{1}{12}$ and $\left\langle z^{4}\right\rangle=\frac{1}{80}$. These limiting values agree with our results at $\kappa=0.5$ and are also approached for larger values of $\kappa$ with increasing size of the lattice.

\section{Monte Carlo results in $4 d$}

Before discussing the results for the $4 \mathrm{~d}$ SU(2) Higgs model, it is necessary to give some details of the programs developed by us to evaluate $K_{t, \mu}$. Because the interpolation in the Seiberg case is done on the original links, and not on the gauge fixed links, the fields tend to be very rough. The same happens if one wants to evaluate the topological charge $q^{w}(n)$ via the section. One of the integrations in eq. (3.12) can be done in analytic form, so we are left with a two-dimensional integral. These integrals must be evaluated carefully, if one wants to extract the periodic structure in the distribution of CS numbers. On the other hand, if we are only interested in the non-integer and therefore gauge-invariant part of $N_{\mathrm{CS}}$ (used in the discussion of baryon number violation) then it suffices to perform several Landau gauge fixing sweeps before doing the integrals. The effect is that $N_{\mathrm{CS}}$ will be shifted by an integer, in most cases into the interval $-\frac{1}{2} \leqslant N_{\mathrm{CS}} \leqslant \frac{1}{2}$. The advantage is that gauge fields on the links become very smooth and the integrals will converge much faster. To define a convergence criterion we note that $K_{t, \mu}$ must change by an integer under a gauge transformation. We took various configurations and monitored $N_{\mathrm{CS}}$, first without and then with a Landau gauge fixing sweep. If $\delta K_{n, \mu}$ was an integer within a relative error of $\epsilon=10^{-4}$, the integrals were accepted.

We have used the following strategy, which turned out to be very efficient. The volume term in eq. (3.12) is the most time-consuming part, so we concentrate on that. Since the Chern-Simons numbers on each time slice are hardly correlated we can use all of them. We perform a Gauss-Legendre integration with $8 \times 8$ points and store the resutls for all the $K_{n, 0}$ 's in each time slice. We then repeat this calculation with $16 \times 16$ points and compare the results for each $K_{n, 0}$. If the relative difference is less than $\epsilon$ the contribution is accepted. Otherwise we collect the $K_{n, 0}$ 's which have not yet converged. In these cells we repeat the integration with $32 \times 32$ points instead, compare with the previous values and eventually repeat the procedure with $64 \times 64$ points. This part can be done very efficiently in vectorized form. At this point only in a few cells the results for $K_{n, 0}$ 's have not 
converged, for these we use a library integration routine with interval adaptation. The typical time for evaluation of $N_{\mathrm{CS}}$ (without any gauge fixing) in a time slice with $6^{3}$ lattice points is roughly 60 seconds on the CRAY-YMP. With gauge fixing this is reduced to about 6 seconds. The entire program runs with a speed of about (150-200) Mflops.

As a test of our programs we calculated the time dependence of $N_{\mathrm{CS}}$ on a $4 \mathrm{~d}$ $\mathrm{SU}(2)$ instanton configuration in the complete axial gauge. This is shown in fig. 2 for an instanton of core size $\rho=2$, on an $8^{3} \times 12$ lattice. On the first time slice the gauge fields are in the vacuum sector with $N_{\mathrm{CS}}=0$. On the last time slice the instanton has mediated the tunneling to the other vacuum sector with $N_{\mathrm{CS}}=1$. We note that in the middle $(t=6)$ one finds $N_{\mathrm{CS}}=\frac{1}{2}$. The corresponding field configuration on this time slice is the analog to the sphaleron in the Higgs models and interpolates between the two vacuums at $t=1$ and $t=12$.

For the SU(2) gauge Higgs model we used the action given in eq. (4.7) with $r=2$. We have chosen to study the structure of the CS number distributions for various values of $\kappa$ at fixed $(\beta, \lambda)=(2.25,0.5)$. For this choice of parameters the phase transition between the symmetric and broken phases is known to occur at $\kappa \simeq 0.27$ [16]. We have performed simulations on a $4^{3} \times 2$ lattice at $\kappa=0.25,0.30$ and 0.40 , i.e. on both sides of the transition line. For each of the $\kappa$-values we have measured $N_{\mathrm{CS}}$ on 2000 gauge field configurations without any gauge fixing. In the left column of fig. 3 we show the CS number distributions. The periodic structure is obvious even for $\kappa$ below $\kappa_{\mathrm{c}}$. With increasing $\kappa$ the CS numbers on many of the configurations are close to an integer, and therefore the gauge field configurations can be interpreted as being close to pure gauge. We checked that $N_{\mathrm{Cs}}$ indeed changed by an integer under a gauge transformation, which confirms the quality of

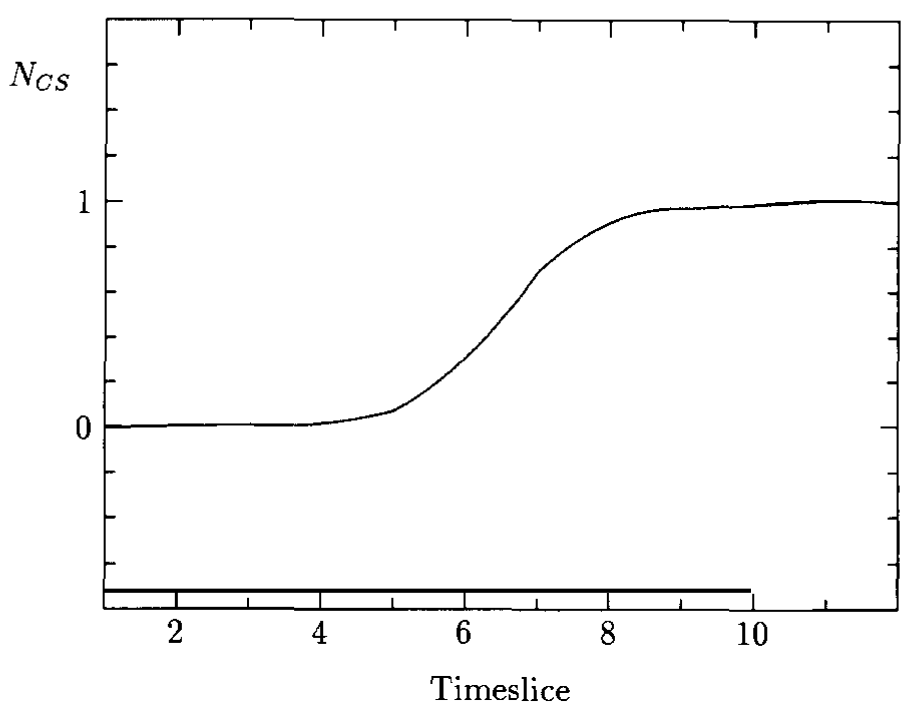

Fig. 2. Profile of $N_{\mathrm{CS}}$ through a $4 \mathrm{~d}$ SU(2) instanton configuration with core size $\rho=2$ on an $8^{3} \times 12$ lattice. 


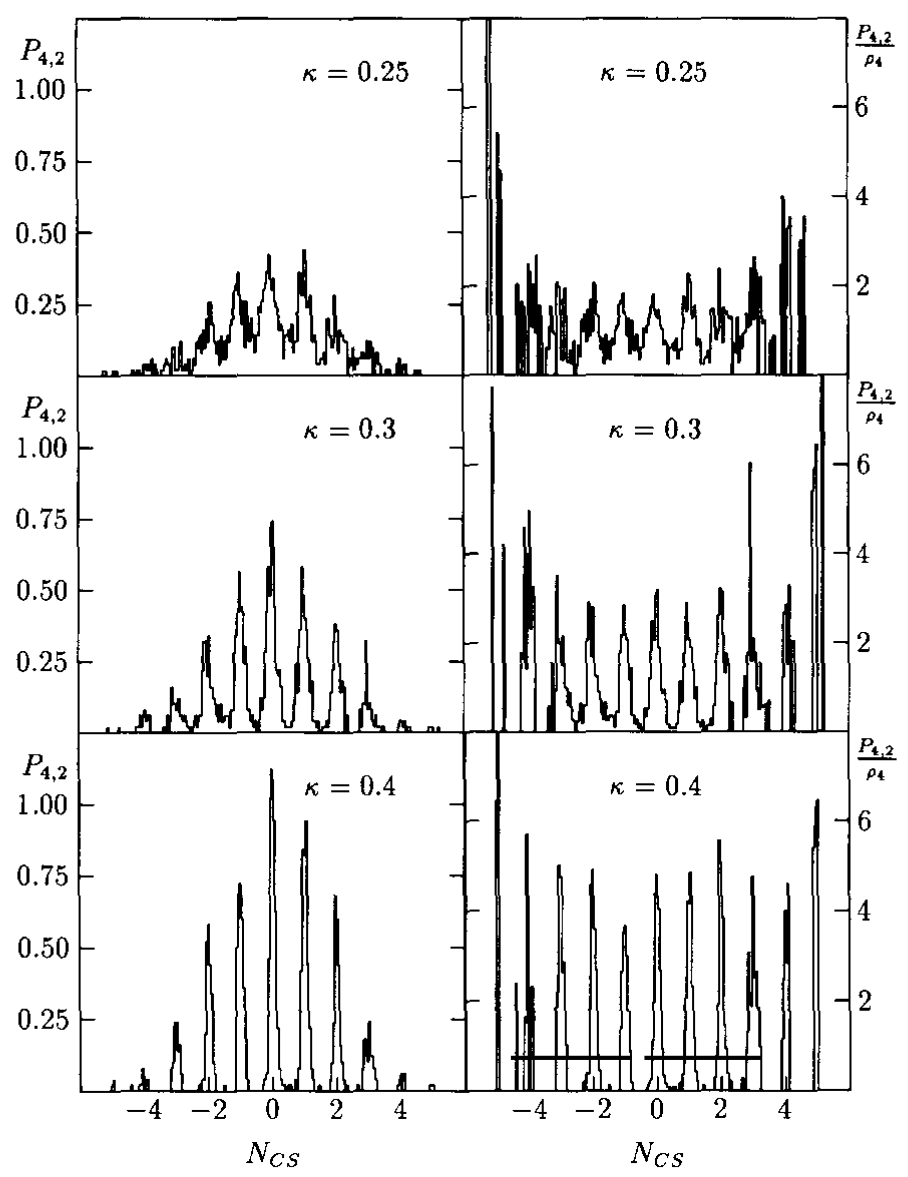

Fig. 3. Probability distributions for Chern-Simons numbers on a $4^{3} \times 2$ lattice. The left column shows the distributions obtained from simulations at $(\beta, \lambda)=(2.25,0.5)$ and the three $\kappa$-values given in the figure. The right column shows the same distributions divided by the strong-coupling distribution, which has been approximated by a gaussian with width $\left\langle N_{\mathrm{CS}}^{2}\right\rangle=2.93$.

our numerical integrations. At $\kappa=0.3$ we have performed axial as well as Landau gauge fixing. Even after a few gauge fixing sweeps nearly all CS numbers get shifted into the interval $\left[-\frac{1}{2}, \frac{1}{2}\right]$. The distribution after only a single gauge fixing sweep is shown in fig. 4 . With a few more Landau gauge fixing sweeps only the peak in the middle would remain.

For the moments of $N_{\mathrm{CS}}$ we find on the $4^{3} \times 2$ lattice at $\kappa=0.30$ the values $\left\langle N_{\mathrm{CS}}^{2}\right\rangle=2.93$ and $\left\langle N_{\mathrm{CS}}^{4}\right\rangle=24.57$. We note that a gaussian fit with width 2.93 would suggest $\left\langle N_{\mathrm{CS}}^{4}\right\rangle=24.96$. Like in two dimensions the moments are thus very close to those of gaussian distributions. Moreover, we also find for the $4 \mathrm{~d} S U(2)$ Higgs model that the moments are independent of $(\beta, \kappa)$ and increase proportional to the spatial volume. We have performed simulations on a $6^{3} \times 2$ lattice (without gauge fixing) at $(\beta, \lambda)=(2.25,0.5)$ and $\kappa=(0.30,0.40)$. For this lattice we find $\left\langle N_{\mathrm{CS}}^{2}\right\rangle=9.63$ and $\left\langle N_{\mathrm{CS}}^{4}\right\rangle=274.39$. A gaussian approximation would lead to $\left\langle N_{\mathrm{CS}}^{4}\right\rangle=275.26$. The general structure of the distributions thus is very similar to the $2 d U(1)$ case and we expect that the finite-size effects can be eliminated 


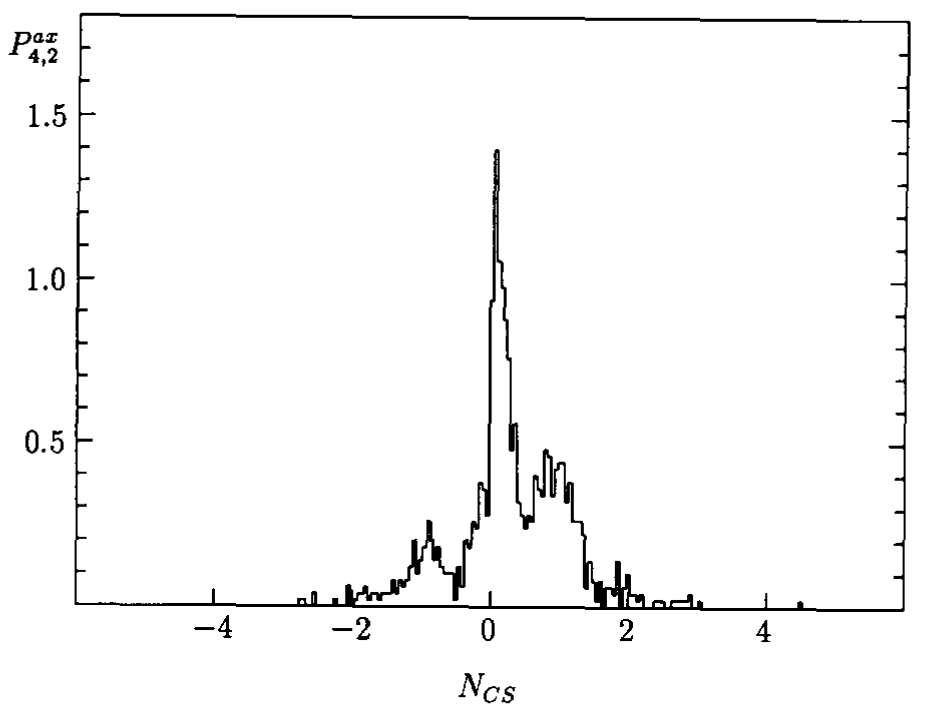

Fig. 4. The Chern-Simons probability distribution on a $4^{3} \times 2$ lattice (one axial gauge fixing sweep) with couplings $(\beta, \lambda)=(2.25,0.5)$ and $\kappa=0.3$.

similarly by dividing out the distribution induced solely by the Haar measure (strong-coupling distribution). We approximate this by a gaussian distribution with the width given by our numerically determined value for $\left\langle N_{\mathrm{CS}}^{2}\right\rangle$. This is shown in the right column of fig. 3. The peaks are now more or less of equal height. We thus may expect, that similar to the $2 \mathrm{~d}$ case the finite-size effects in the distributions drop out in the distribution functions $\omega_{n_{r}, n_{\tau}}(z)$ for the gauge-invariant, non-integer part, $z$, defined in eq. (4.8). We note that these can be calculated on gauge fixed configurations, which is computationally much less demanding.

At finite temperature the tunneling between topologically distinct vacuums should become more likely with increasing temperature. This should be reflected in a flattening of the distributions, $\omega_{n_{\sigma}, n_{\tau}}(z)$, defined in eq. (4.8). In order to see, whether this effect is visible in our distributions, we have performed simulations on lattices of size $6^{3} \times n_{\tau}$ with $n_{\tau}=2,4$ and 6 . For these lattices we used 50 Landau gauge fixing sweeps, to make the integrals converge fast. For both lattice sizes we collected 6000 Chern-Simons numbers at $\kappa=0.3$. The results for $\omega_{n_{o}, n_{\tau}}(z)$ are shown in fig. 5 . The left column shows the change in the distributions with varying $n_{\tau}$ at fixed $n_{\sigma}$. The tendency for a flattening of the distribution at finite temperature is clearly visible. We take this as evidence that the system tunnels more often at non-zero temperature.

A major problem for a more quantitative analysis of the distributions at finite temperature is caused by the volume dependence of $\omega_{n_{\sigma}, n_{\tau}}(z)$ itself, which leads to a flattening of the distributions with increasing spatial volume. This is seen in the right column of fig. 5, where we compare distributions on $4^{3} \times 2,6^{3} \times 2$ and $8^{3} \times 2$ lattices. Clearly the distributions become flatter with increasing spatial lattice size. We note, that this effect, caused by the spatial volume, is opposite to the 

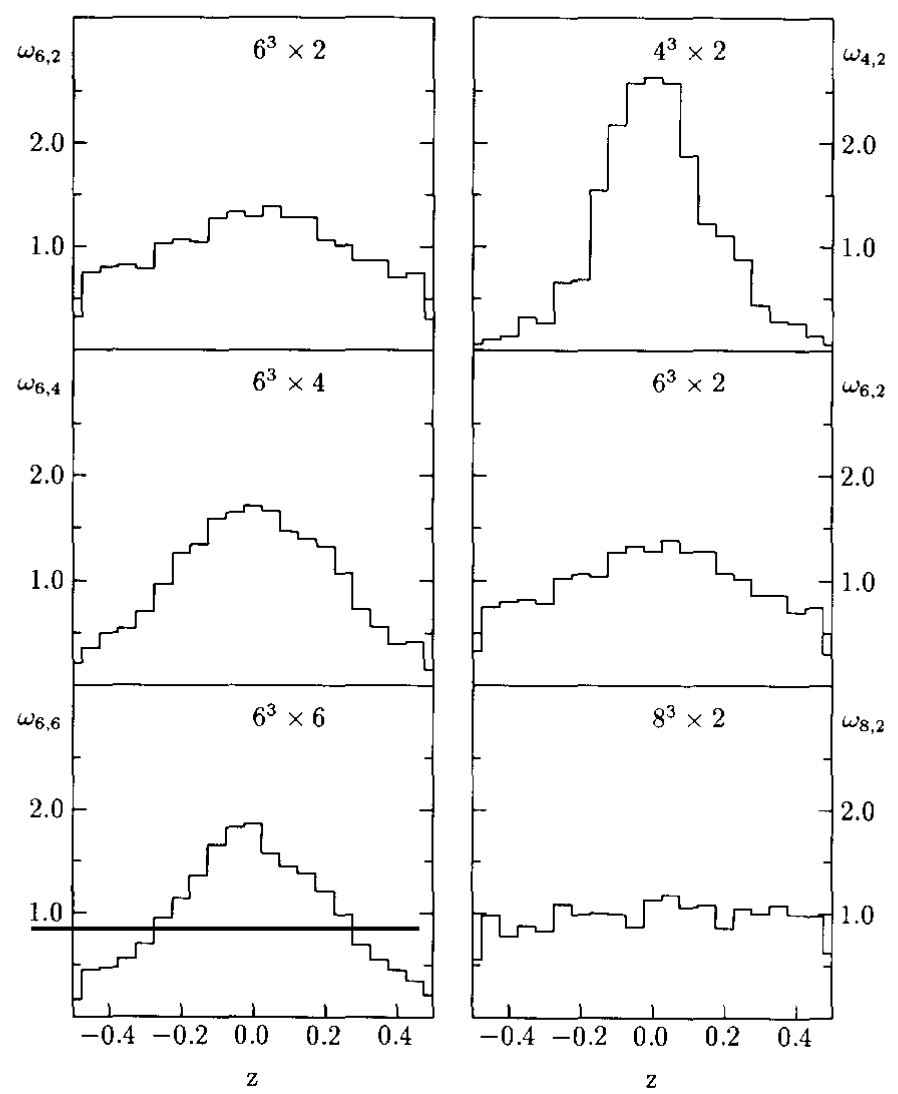

Fig. 5. Probability distributions, $\omega_{n, n}$, for Chern-Simons numbers calculated on lattices of size $n_{\sigma}^{3} \times n_{\tau}$ and fixed couplings $(\beta, \lambda, \kappa)=(2.25,0.5,0.3)$. The left column shows the distributions obtained from simulations at constant spatial volume, and the right column shows the distributions for increasing spatial volume at constant temperature $\left(n_{\tau}\right)$.

finite-temperature effect shown in the left column of fig. 5. In that case the distributions become more peaked around $N_{\mathrm{CS}}=0$, although the total 4-volume increases.

TABLE 3

The width of the distribution of the non-integer part $(z)$ of Chern-Simons numbers, $N_{\mathrm{CS}}=m+z$, for the $4 \mathrm{~d}$ SU(2) Higgs model on lattices of size $n_{\sigma}^{3} \times n_{\tau}$ at $(\beta, \lambda)=(2.25,0.5)$ and various values of $\kappa$

\begin{tabular}{cccc}
\hline$N_{\sigma}$ & $N_{\tau}$ & $\kappa$ & $\left\langle z^{2}\right\rangle$ \\
\hline 4 & 2 & 0.30 & $0.028(2)$ \\
6 & 2 & 0.30 & $0.068(2)$ \\
8 & 2 & 0.30 & $0.083(1)$ \\
6 & 2 & 0.30 & $0.068(2)$ \\
6 & 4 & 0.30 & $0.049(2)$ \\
6 & 6 & 0.30 & $0.049(2)$ \\
4 & 2 & 0.25 & $0.057(2)$ \\
4 & 2 & 0.30 & $0.028(2)$ \\
4 & 2 & 0.40 & $0.009(2)$ \\
\hline
\end{tabular}


The moments, $\left\langle z^{2}\right\rangle$, of the distributions shown in fig. 5 are summarized in table 3. They show that the limiting value for a flat distribution, $\left\langle z^{2}\right\rangle=\frac{1}{12}$ is rapidly approached with increasing spatial volume as well as decreasing couplings $\beta$ and/or $\kappa$. As mentioned before the broadening of the distribution with increasing spatial volume is not unexpected. It has been shown [6] that even at zero temperature quantum fluctuations of the fields can lead to large values of $N_{\mathrm{CS}}$. In fact, this contribution is proportional to the spatial volume. In the present parameter range studied by us such a linear dependence is not visible. We will have to work at much larger values of the couplings $\beta$ and/or $\kappa$. This will be necessary in order to proceed with a quantitative analysis of finite-temperature effects on the CS number distributions.

\section{Conclusions}

We have studied the properties of Chern-Simon numbers in two- and four-dimensional Higgs models on euclidean lattices. We have shown that the definition of the Chern-Simons term based on the geometric definition of topological charges preserves the basic properties of the continuum expressions. The non-integer part of the Chern-Simons number is gauge invariant; the Chern-Simons number changes by an integer under gauge transformations. Moreover, we have shown that the effective potential for the Chern-Simons number is periodic with maxima of equal height at integer values of $N_{\mathrm{CS}}$, if finite-size effects are taken into account properly.

The distribution of Chern-Simon numbers flattens with increasing temperature. This will lead to an increase of baryon number violating processes at high temperature. At present we could, however, not explore the temperature dependence of the transition rates quantitatively as the distributions are still influenced by finite-size effects caused by quantum fluctuations in the trivial vacuum. These fluctuations will be suppressed at larger values of the gauge coupling, which we might have to choose for a future quantitative analysis of the Chern-Simons number distributions in the vicinity of the symmetry-restoring phase transition of the 4d SU(2) Higgs model [17]. Another possibility to suppress the contribution from vacuum fluctuation is based on a modification of the cooling method, which also allows one to smoothen configurations and obtain the contribution of classical configurations that extremize the euclidean action [18]. This approach has recently been used in the context of the $2 \mathrm{~d} \mathrm{O}(3)$ non-linear sigma model [19] and is in an gauge-invariant manner also applicable to SU(2) gauge theories [20]. It will be interesting to test with our geometric formulation whether the Chern-Simons numbers develop a plateau under extremization of the euclidean action. This would allow one to extract the distribution of Chern-Simons numbers on topologically non-trivial gauge field configurations for which the quantum fluctuations are suppressed. 
The numerical simulations described here have been performed on the CrayYMP at HLRZ. Financial support from DFG under contract Pe 340/1-3 and the Ministerium für Wissenschaft und Forschung NRW under contract IVA5-10600990 is gratefully acknowledged.

\section{Appendix}

We will first show that the following identities are true on lattices with spatial extent $(2 n+1)$ and $2 n$, respectively:

$$
\begin{gathered}
\sum_{m=-n}^{n} \rho_{2 n+1}(z+m)=1, \quad|z| \leqslant \frac{1}{2} . \\
\sum_{m=-n}^{n-1} \rho_{2 n}(z+m)=1, \quad 0<z \leqslant 1 .
\end{gathered}
$$

One has

$$
\begin{aligned}
(2 n) ! \sum_{m=-n}^{n} \rho_{2 n+1}(z+m)= & \sum_{m=1}^{n} \sum_{i=0}^{n-m}(-1)^{i}\left(\begin{array}{c}
2 n+1 \\
i
\end{array}\right)\left(n+\frac{1}{2}-i-z-m\right)^{2 n} \\
& +\sum_{m=1}^{n} \sum_{i=0}^{n-m}(-1)^{i}\left(\begin{array}{c}
2 n+1 \\
i
\end{array}\right)\left(n+\frac{1}{2}-i+z-m\right)^{2 n} \\
& +\sum_{i=0}^{n}(-1)^{i}\left(\begin{array}{c}
2 n+1 \\
i
\end{array}\right)\left(n+\frac{1}{2}-i-|z|\right)^{2 n}
\end{aligned}
$$

Interchanging the summation over $(i, m)$ in the double sum gives for the first term

$$
\begin{gathered}
\sum_{m=1}^{n} \sum_{i=0}^{n-m}(-1)^{i}\left(\begin{array}{c}
2 n+1 \\
i
\end{array}\right)\left(n+\frac{1}{2}-i-z-m\right)^{2 n} \\
\quad=\sum_{i=0}^{n-1} \sum_{m=0}^{i}(-1)^{m}\left(\begin{array}{c}
2 n+1 \\
m
\end{array}\right)\left(n-\frac{1}{2}-i-z\right)^{2 n} .
\end{gathered}
$$

The summation over $m$ can now be performed giving for the first term in eq. (A.2)

$$
\sum_{i=0}^{n-1}(-1)^{i}\left(\begin{array}{c}
2 n \\
i
\end{array}\right)\left(n-\frac{1}{2}-i-z\right)^{2 n}
$$


Using now also the recursion relation,

$$
\left(\begin{array}{c}
2 n+1 \\
i
\end{array}\right)=\left(\begin{array}{c}
2 n \\
i
\end{array}\right)+\left(\begin{array}{c}
2 n \\
i-1
\end{array}\right)
$$

in the third term of eq. (A.2), we obtain

$$
(2 n) ! \sum_{m=-n}^{n} \rho_{2 n-1}(z+m)=\sum_{i=0}^{2 n}(-1)^{i}\left(\begin{array}{c}
2 n \\
i
\end{array}\right)\left(n-\frac{1}{2}-i+z\right)^{2 n}=(2 n) !
$$

This concludes the proof.

Let us now calculate the moments of this distribution. We consider the integrals

$$
\begin{aligned}
\mathscr{P}_{2 n}^{\alpha} & =\int_{-n}^{n} \mathrm{~d} z z^{\alpha} \rho_{2 n} \\
& =\frac{2}{(2 n-1) !} \sum_{m=0}^{n-1} \sum_{i=0}^{n-1-m}(-1)^{i}\left(\begin{array}{c}
2 n \\
i
\end{array}\right) \int_{m}^{m+1} \mathrm{~d} z z^{\alpha}(n-i-|z|)^{2 n-1}
\end{aligned}
$$

Interchanging the summation over $(i, m)$ in the double sum and then integrating over $z$ yields

$$
\mathscr{P}_{2 n}^{\alpha}=\frac{\alpha !}{(2 n+\alpha) !} \sum_{i=0}^{2 n}(-1)^{i}\left(\begin{array}{c}
2 n \\
i
\end{array}\right)(n-i)^{2 n+\alpha}
$$

The Stirling numbers of the second kind,

$$
\mathscr{S}_{n}^{m}=\frac{(-1)^{m}}{m !} \sum_{i=0}^{m}(-1)^{i}\left(\begin{array}{c}
m \\
i
\end{array}\right) i^{n}
$$

with the recursion

$$
\mathscr{S}_{n}^{m}=\sum_{i=1}^{m} i \mathscr{S}_{i+n-m-1}^{i}, \quad \mathscr{S}_{m}^{m}=1
$$

can be used to write the moments as

$$
\mathscr{P}_{2 n}^{\alpha}=\frac{(2 n) ! \alpha !}{(2 n+\alpha) !} \sum_{i=0}^{\alpha}(-n)^{\alpha-i}\left(\begin{array}{c}
2 n+\alpha \\
\alpha-i
\end{array}\right) \mathscr{S}_{2 n+i}^{2 n}
$$


In the following we shall only consider the cases $\alpha=(0,1,2,3,4)$. From the recursion formula it follows easily:

$$
\begin{aligned}
\mathscr{S}_{m+1}^{m} & =\left(\begin{array}{c}
m+1 \\
2
\end{array}\right), \\
\mathscr{S}_{m+2}^{m} & =\frac{3 m+1}{4}\left(\begin{array}{c}
m+2 \\
3
\end{array}\right), \\
\mathscr{S}_{m+3}^{m} & =\frac{m(m+1)}{2}\left(\begin{array}{c}
m+3 \\
4
\end{array}\right), \\
\mathscr{S}_{m+4}^{m} & =\frac{15 m^{3}+30 m^{2}+5 m-2}{48}\left(\begin{array}{c}
m+4 \\
5
\end{array}\right) .
\end{aligned}
$$

This leads to

$$
\begin{aligned}
& \mathscr{P}_{2 n}^{0}=1, \\
& \mathscr{P}_{2 n}^{1}=0, \\
& \mathscr{P}_{2 n}^{2}=\frac{n}{6}, \\
& \mathscr{P}_{2 n}^{3}=0, \\
& \mathscr{P}_{2 n}^{4}=-\frac{n}{60}+\frac{n^{2}}{12} .
\end{aligned}
$$

We also have checked that the following relations for the "discrete" momenta hold as long as the degree of the moment does not exceed the lattice size:

$$
\begin{gathered}
\sum_{m=-n}^{n}(z+m)^{k} \rho_{2 n+1}(z+m)=\mathscr{P}_{2 n+1}^{k}, \quad k<2 n+1, \quad|z| \leqslant \frac{1}{2} . \\
\sum_{m=-n}^{n-1}(z+m)^{k} \rho_{2 n}(z+m)=\mathscr{P}_{2 n}^{k}, \quad k<2 n, \quad 0<z \leqslant 1 .
\end{gathered}
$$

\section{References}

[1] G. Rajaraman, Solitons and instantons (North-Holland, Amsterdam, 1982)

[2] F. Klinkhamer and N. Manton, Phys. Rev. D14 (1976) 3432

[3] G. 't Hooft, Phys. Rev. Lett. 37 (1976) 8; Phys. Rev. D30 (1984) 2212 
[4] D.Yu. Grigoriev, V.A. Rubakov and M.E. Shaposhnikov, Phys. Lett. B216 (1989) 172; Nucl. Phys. B326 (1989) 737;

A.I. Bochkarev and Ph. de Forcrand, Phys. Rev. D44 (1991) 519; IPS-92-18 preprint;

M. Alford, H. Feldman and M. Gleiser, Phys. Rev. Lett. 68 (1992) 1645; NFS-ITP-92-91 preprint

[5] J. Ambjørn, M.L. Laursen and M.E. Shaposhnikov, Phys. Lett. B197 (1987) 49; Nucl. Phys. B316 (1989) 483

[6] J. Ambjørn, T. Asgaard, H. Porter and M.E. Shaposhnikov, Phys. Lett. B244 (1990) 479

[7] J. Ambjørn and K. Farakos, Niels-Bohr Preprint, NBI-HE-92-52

[8] F. Karsch, M.L. Laursen, T. Neuhaus, B. Plache and U.-J. Wiese, Int. J. Mod. Phys. C3 (1992) 39; F. Karsch, M.L. Laursen, T. Neuhaus and B. Plache, in Proc. Int. Symp. Lattice 92, 15-19 September 1992, Amsterdam, The Netherlands, Nucl. Phys. B (Proc. Suppl.) 30 (1993) 715

[9] M. Lüscher, Commun. Math. Phys. 85 (1982) 39

[10] N. Seiberg, Phys. Lett. B148 (1984) 456

[11] I.A. Fox, J.P. Gilchrist, M.L. Laursen and G. Schierholz, Phys. Rev. Lett. 54 (1985) 749

[12] M. Göckeler, A.S. Kronfeld, G. Schierholz and U.-J. Wiese, HLRZ preprint, HLRZ-92-34; A. Phillips and D. Stone, Nucl. Phys. B (Proc. Suppl.) 20 (1991) 28

[13] M. Göckeler, A.S. Kronfeld, M.L. Laursen, G. Schierholz and U.-J. Wiese, Nucl. Phys. B292 (1987) 349

[14] M.L. Laursen, HLRZ preprint, HLRZ-92-61

[15] U.-J. Wiese, Nucl. Phys. B318 (1989) 153

[16] W. Bock, H.G. Evertz, J. Jersak, D.P. Landau, T. Neuhaus and J.L. Xu, Phys. Rev. D41 (1990) 2573

[17] B. Bunk, E.M. Ilgenfritz, J. Kripfganz and A. Schiller, Phys. Lett. B284 (1992) 371; Bielefeld preprint, BI-TP 92/46;

H.G. Evertz, Ph.D. thesis, University of Aachen, Germany, 1987

[18] A. Duncan and R.D. Mawhinney, Phys. Lett. B282 (1992) 423

[19] C. Michael and J. Kripfganz, Liverpool preprint, LTH 298 (1993)

[20] A.J. van der Sijs, Phys. Lett. B294 (1992) 391 